\title{
Aspectos psicossociais e intervenções realizadas com o adolescente depressivo: revisão integrativa nacional da última década
}

\author{
Psychosocial aspects and interventions realized depression in adolescence: \\ integrative review of the last decade
}

\section{Gerusa Marcondes Pimentel de Abreu Lima', João Vítor Rodrigues Mioº, Graciella Navarros Santos ${ }^{3}$, Claudinei José Gomes Campos ${ }^{4}$}

'Autora para correspondência. Universidade Estadual de Campinas, Campinas, São Paulo, Brasil. ORCID: 0000-0001-8690-4948. gerusa.abreulima@gmail.com ${ }^{2}$ Universidade Estadual de Campinas, Campinas, São Paulo, Brasil. ORCID: 0000-0002-0295-3011. joaovitormio@yahoo.com.br 3Universidade Estadual de Campinas, Campinas, São Paulo, Brasil. ORCID: 0000-0002-3158-2141.nvarros@hotmail.com ${ }^{4}$ Universidade Estadual de Campinas, Campinas, São Paulo, Brasil. ORCID: 0000-0001-9587-6694.ccampos@unicamp.br

RESUMO I INTRODUÇÃO: Somente no inicio da década de 70 que a depressão na adolescência foi conceituada uma doença, visto a julgar que este transtorno ocasiona danos e prejúzos considerados de alta complexidade. O objetivo do estudo foi analisar as produções cientificas da literatura brasileira na última década, a fim de aprofundar o conhecimento sobre o transtorno depressivo em adolescentes. MÉTODO: a presente investigação é uma revisão integrativa nacional, compreendida no ano de 2007 a 2016, integrados no seguintes banco de dados: Scielo, LILACS, Scopus, CINAHL, PUBMED, MEDLINE, PUBMED e BDEnf. A análise temática foi usada para a discussão dos 15 artigos encontrados. RESULTADOS: Os comportamentos negativos nas relações sociais, principalmente, depressão, ansiedade e agressividade, costumam ficar evidenciados em torno dos 11 a 16 anos de idade. CONSIDERAÇÕES FINAIS: O meio e a mídia social em que o adolescente está inserido é considerado um fator de impacto que reflete não somente no âmbito familiar, mas também, na escola e na sociedade em geral.

PALAVRAS-CHAVE: Adolescente. Depressão. Poder familiar. Terapêutica.

\begin{abstract}
BACKGROUND: Depression in adolescence, since it is a disease in the 1970s. It is considered that this disorder causes damages and losses of high complexity. OBJECTIVE: The study was to analyze national scientific productions to comprehend the factors associated with depression adolescents. METHODOLOGY: review of national integrative literature of scientific researches, comprehended between 2007 and 2016, integrated on the following databases: Scielo, LILACS, Scopus, CINAHL, PUBMED, MEDLINE and BDEnf. The guiding question was: what are the psychosocial factors associated to depressive adolescents.Thematic analysis was utilized to data discussion 15 articles. RESULTS: The negative behaviors on social relations, disorders of internalizing and externalizing types, mainly depression, anxiety and aggressiveness are usually evident around 11 to 16 years old. CONCLUSION: The social environment and media marketing where the adolescent is inserted is considered an impact factor that reflects not only the family circle, but also the school and the society in general.
\end{abstract}

KEYWORDS: Depression. Adolescent. Parenting. Therapeutics. 


\section{Introdução}

A adolescência é um período de grande importância para a construção do indivíduo, onde há diversas mudanças, sejam elas orgânicas, físicas, mentais e comportamentais (World Health Organization, 2017). A depressão na adolescência, somente foi considerada uma doença nessa idade na década de 1970, pois até o momento acreditava-se que esses jovens não desenvolviam a depressão ou ainda, o transtorno era confundido com apenas uma fase do processo do desenvolvimento o qual o adolescente passava. No entanto, pesquisadores e estudiosos afirmaram que o transtorno depressivo pode estar presente em todas as faixas etárias (Crujo \& Marques, 2009).

Os sintomas comumente apresentados nos adolescentes depressivos são: humor deprimido, desinteresse, oscilações de emoções e alguns sintomas de angústia e de ansiedade; os sintomas cognitivos, como a diminuição de energia e falhas na memória e na concentração, também são critérios de avaliação e sofrem danos causados pela doença (Chavez, 2014). Além disso, o uso de substâncias ainda esteve diretamente ligado com a sintomatologia depressiva, sendo o uso de tabaco, maconha e álcool (Rojas et al., 2012).

O transtorno depressivo igualmente está associado com as dificuldades e obstáculos socioeconômicos, podendo desencadear desequilíbrio familiar, assim, as consequências dessas oscilações são: separação dos pais, a falta de comunicação intrapessoal, violência doméstica entre outras. Situações como essas de desarmonia familiar geram elementos que devem ser analisados, visto que são fontes significativas e propulsoras ao desencadeamento do transtorno depressivo nos adolescentes (Xerinda \& Maluschke, 2015). Ainda, há estudos indicando que a depressão na fase adulta pode ter tido o seu iniciona adolescência (Resende, Santos, Santos, \& Ferrão, 2013).
A discussão do gênero foi alvo de estudo retrospectivo realizado com crianças e adolescentes por Encarnação, Moura, Gomes e Silva (2011) que observaram à prevalência do sexo masculino relacionado à síndrome depressiva.

Convém referenciar que a busca do atendimento especializado, evidenciou que as figuras parentais bem como, os cuidadores responsáveis pelos adolescentes são essências para um diagnóstico precoce, pois a percepção dos pais possui um caráter interventivo frente ao tratamento (Antunes, Campos, Lima \& Ferraz, 2016). Considera-se que a depressão desenvolvida no adolescente por si só, ocasiona danos e prejuízos considerados de alta complexidade à vida desses sujeitos, não necessariamente estando relacionados ou associados à outras doença e comorbidades. (Melo, Siebra, \& Moreira, 2017). Contudo, o presente estudo teve como objetivo principal, realizar uma revisão integrativa da literatura brasileira, a fim de compreender os fatores associados à depressão e as intervenções recebidas por esses adolescentes com o transtorno.

\section{Metodologia}

Considera-se que uma revisão integrativa, utilizada no presente estudo, tem como objetivo primordial a análise criteriosa da literatura, que reúne um número significativo de manuscritos publicados, com a finalidade de responder ou mesmo buscar informações a uma pergunta gerada, assim, tem como objetivo o encontro da compreensão em profundidade sobre o tema pesquisado, levando a novas indagações e desvelamento dos resultados descoberto (Whittemore \& Knalt, 2005).

O trabalho com a revisão integrativa necessita de alguns critérios que devem ser seguidos, tais como, deixar explícito o tema e o objetivo que está sendo 
investigado; formulação precisa da pergunta norteadora; definir os critérios de inclusão e exclusão que comporão as amostras dos artigos selecionados na literatura e base de dados, definição dos documentos a serem pesquisados nos artigos resgatados; avaliar os manuscritos encontrados e explanar os dados descobertos (Souza, Silva, \& Carvalho, 2010; Sobral \& Campos, 2012)

A pergunta norteadora para a revisão desse estudo foram: "Quais são os fatores psicossociais associados aos adolescentes depressivos"? E quais são as intervenções recebidas por esses adolescentes?

A busca na base de dados teve início em novembro de 2016 a janeiro de 2017, foram pesquisados: a Scielo, LILACS, Scopus, CINAHL, PUBMED, MEDLINE, PUBMED e BDEnf . Os critérios de inclusão para a seleção dos artigos foram abarcados da seguinte forma: deveriam ser artigos originais e completos produzidos entre 2007 a 2016, na língua portuguesa e realizado por pesquisadores nacionais. $E$ abranger nesta busca, somente pesquisas com sujeitos adolescentes e relacionados com o transtorno depressivo. No entanto, necessitariam responder a pergunta norteadora. As palavras empregadas na procura pelos manuscritos estavam relacionadas nos descritores em Ciência da Saúde (DECS) e Medicais Subject Headings (MESH), dessa forma, foram usados os seguintes termos, depressão, adolescente e tratamento, foi utilizado o boleano "AND" para re- alizar a relação dos termos. A aplicação para os critérios de exclusão se deu pelos manuscritos indexados repetidos e ou duplicados nas bases de dados, editoriais, artigo de revisão, literatura cinzenta e sistemática, internacionais e que não correspondia ao objetivo proposto. Destacamos que os autores realizaram leitura exaustiva e independente dos artigos para o procedimento de inclusão e exclusão. Os procedimentos de busca empregados nas respectivas bases de dados e as razões da exclusão foram apontados na Quadro 1, como proposto pelos critérios do PRISMA

Ressalta-se que tais ações foram verificadas e analisadas por dois revisores autônomos, sendo que um terceiro estava disponível no caso de discordâncias sobre a inclusão ou não de estudos.

\section{Resultados}

A amostra da busca compreendeu-se de 674 artigos encontrados inicialmente, após uma exaustiva leitura a princípio dos resumos e depois de aplicado os critérios de exclusão e inclusão acima, foram excluídos 660 artigos. Dessa forma, realizou-se a leitura completa de cada artigo pelos autores de forma individual, para a verificação do presente objetivo do estudo, assim, se obteve ao final do processo de avaliação, 15 artigos selecionados.

Quadro 1. Artigos recuperados no banco de dados

\begin{tabular}{|c|c|c|c|}
\hline Base de Dados & Artigos recuperados & $\begin{array}{c}\text { Artigos excluídos } \\
\text { Após os critérios de } \\
\text { exclusão }\end{array}$ & Artigos selecionados \\
\hline Scielo & 39 & 35 & 5 \\
\hline Lilacs & 350 & 345 & 5 \\
\hline Scopus & 198 & 194 & 4 \\
\hline Embase & 17 & 17 & 0 \\
\hline MedLine & 46 & 46 & 0 \\
\hline CINAHL & 18 & 18 & 1 \\
\hline BDEnf & 06 & 05 & 15 \\
\hline Total & 674 & 660 & 0 \\
\hline
\end{tabular}


Abaixo, a Tabela 1 retrata a classificação dos achados, em relação à base de dados,

Tabela 1. Distribuição dos artigos segundo autor e indexadores

\begin{tabular}{|c|c|c|c|}
\hline Autores & Indexador & Autores & Indexador \\
\hline $\begin{array}{l}\text { El.Fukuda, C. C., Penso, M. A., Amparo, } \\
\text { D. M., Almeida, B. C., \& Morais, C. A. } \\
\text { (2016). Saúde mental de jovens } \\
\text { brasileiros: barreiras à busca por ajuda } \\
\text { profissional. Estudos de Psicologia, } \\
33(2), 355-365 \text {. }\end{array}$ & Scopus. & $\begin{array}{l}\text { E2.Crivelatti, M. M. B., Durman, } \\
\text { S., \& Hofstatter, L. M. (2007). } \\
\text { Sofrimento Psíquico na } \\
\text { Adolescência. Contexto Enferm, } \\
15,64-70 .\end{array}$ & Lilacs. \\
\hline $\begin{array}{l}\text { E3.Monteiro, K.C.C. \& Lage, A.M.V. } \\
\text { (2007). A Depressão na Adolescência. } \\
\text { Psicologia em Estudo, 12(2), 257- } \\
\text { 265. doi: } 10.1590 / \text { S1 } 413 \text { - } \\
\text { 73722007000200006 }\end{array}$ & Lilacs. & $\begin{array}{l}\text { E4.Avanci, J. Q., Assis, S. G., \& } \\
\text { Oliveira, R. V. C. } \\
\text { (2008).Sintomas depressivos na } \\
\text { adolescência: estudo sobre } \\
\text { fatores psicossociais em amostra } \\
\text { de escolas de um município do } \\
\text { Rio de Janeiro. Cad. Saúde } \\
\text { Pública,24(10),2334-2346. } \\
\end{array}$ & Scielo. \\
\hline $\begin{array}{l}\text { E5.Antunes, H.M., \& Campos, C.J.G. } \\
\text { (2007). Pais e responsáveis do } \\
\text { adolescente deprimido: buscando } \\
\text { conhecer experiências que levaram à } \\
\text { procura de atendimento } \\
\text { especializado. Rev Esc Enferm USP, } \\
41(2), 205-212 \text {. doi: } \\
\quad 10.1590 / \text { S0080- } \\
62342007000200005\end{array}$ & BDEnf. & $\begin{array}{l}\text { E6.Teodoro, M. L. M., Cardoso, } \\
\text { B. M. \& Freitas A. C. H. (2009). } \\
\text { Afetividade e Conflito familiar e } \\
\text { sua Relação com a Depressão } \\
\text { em Crianças e Adolescentes. } \\
\text { Psicologia: Reflexão e Crítica, } \\
\text { 23(2), 324-333. }\end{array}$ & Scielo \\
\hline $\begin{array}{l}\text { E7.Melo,A.K.S\& Moreira, V. (2008). } \\
\text { Fenomenologia da queixa depressiva em } \\
\text { adolescentes: um estudo crítico-social. } \\
\text { Aletheia, 27(1), } 51-64\end{array}$ & Lilacs. & $\begin{array}{l}\text { E8.Valverde, B.S.C.L., Vitalle, } \\
\text { M.S.S., Sampaio, I.P.C., \& } \\
\text { Schoen, T.H. (2012) } \\
\text { Levantamento de Problemas } \\
\text { Comportamentais/ Emocionais } \\
\text { em um Ambulatório para } \\
\text { Adolescentes. Paideia,22(53), } \\
315-323 .\end{array}$ & Lilacs. \\
\hline \begin{tabular}{|} 
E9.Aragão, T.A., Coutinho, M.P.L., Araújo, \\
L.F. \& Castanha, A.R. (2009). Uma \\
perspectiva psicossocial da \\
sintomatologia depressiva na \\
adolescência. Ciência e Saúde \\
Coletiva, 14(2), 395-405. doi: \\
$10.1590 /$ S1413-81232009000200009 \\
\end{tabular} & Lilacs & $\begin{array}{l}\text { E10.Jatobá, J. D. V. N. \& Bastos, } \\
\text { O. (2007). Depressão e } \\
\text { ansiedade em adolescentes de } \\
\text { escolas públicas e privadas. J } \\
\text { Bras Psiquiatr, 56(3), 171-179. }\end{array}$ & Scielo. \\
\hline $\begin{array}{l}\text { E1 1.Moutinho, A. I. G., Oliveira, A. R. F., } \\
\text { Torres, B. D. P. S. A., Costa, N. R. G., } \\
\text { Brito, S. M. D., \& Azevedo, S. M. (2013). } \\
\text { E-DARS - Estudo da depressão em } \\
\quad \text { adolescentes e rede socias. } \\
\text { Adolesc. Saúde, 10(2), 30-38. }\end{array}$ & Scopus & $\begin{array}{l}\text { E1 2.Abaid, J. L. W., Aglio, D. D. } \\
\text { D., \& Koller, S. H. } \\
\text { (2010).Preditores de sintomas } \\
\text { depressivos em crianças e } \\
\text { adolescentes institucionalizados. } \\
\text { Universidade Psychol, 9(1), } \\
199-212 .\end{array}$ & Scopus \\
\hline $\begin{array}{l}\text { E13.Barbosa, D. G., Andrade, R. D., } \\
\text { Teixeira, C. S., Neto, M. G. F., \& Felden, } \\
\text { E. P. G. (2016). Sintomas depressivos em } \\
\text { adolescentes em situação de } \\
\text { vulnerabilidade social. Cad. Saúde } \\
\text { Colet. doi: } 10.1590 / 1414- \\
\text { 462X201600020195. }\end{array}$ & Scielo. & $\begin{array}{l}\text { E1 4.Souza, L. D. M., Silva R. S., } \\
\text { Godoy R. V., Cruzeiro. A. L. S., } \\
\text { Faria, A. D., Pinheiro R. T., } \\
\text { Horta, B. L., \& Silvia, R. A. } \\
\text { (2008). Sintomatologia } \\
\text { depressiva em adolescentes } \\
\text { iniciais - estudo de base } \\
\text { populacional. J Bras Psiquiatr, } \\
\text { 57(4), 261- } \quad 266 .\end{array}$ & Scopus. \\
\hline $\begin{array}{l}\text { El 5.Ribeiro, K.C.S.,Coutinho, M.P.L.\& } \\
\text { Nascimento, E.S. (2010). Representação } \\
\text { social da depressão em uma Instituição } \\
\text { de Ensino da Rede Pública. Psicologia: } \\
\text { Ciência e Profissão, 30(3), 448- }\end{array}$ & Scielo & & \\
\hline
\end{tabular}


Optamos para tratamento dos dados dos artigos nomeados, a análise de conteúdo de Bardin (2011), visto que a análise de conteúdo é uma técnica de levantamento de dados como procedimento de análise de dados, que permitirá a compreensão para codificar a unidade de registro, ou seja, visando a compreensão, interpretação e sentido das unidades de registros trabalhadas, com a finalidade de sistematizar de tal modo, as duas categorias temáticas que surgiram: fatores emocionais e psicossociais de adolescentes depressivos e tratamento recebido antes e depois do atendimento médico.

Tais categorias serão descritas a seguir. Sendo utilizados critérios de concordância entre os autores que avaliaram separadamente o material extraído da revisão de literatura, E após as análises individuais foi realizada uma reunião para evidenciar e explorar os temas advindos do parecer final dos pares. Abaixo representado no Quadro 2. As categorias e suas temáticas desenvolvidas nos achados da presente revisão.

Quadro 2. Categorias e suas temáticas desenvolvidas na análise de dados

\begin{tabular}{|l|l|}
\hline \multicolumn{2}{|c|}{ CATEGORIAS } \\
\hline $\begin{array}{l}\text { Fatores emocionais e psicossociais de } \\
\text { adolescentes depressivos }\end{array}$ & $\begin{array}{l}\text { Intervenções recebidas: antes e depois do } \\
\text { tratamento ambulatorial }\end{array}$ \\
\hline & TEMÁTICA \\
\hline Sentimentos dos adolescentes & Relação médico e paciente \\
\hline Rede Social & Apoio do cuidador \\
\hline Escola & Tratamento farmacológico \\
\hline Vulnerabilidade emocional & Tratamento psicoterapêutico \\
\hline Vulnerabilidade social & Saúde mental \\
\hline Mídia e internet & Busca pelo tratamento \\
\hline Substâncias psicoativas & \\
\hline
\end{tabular}

\section{Discussão}

\section{Fatores emocionais e psicossociais de adolescen- tes depressivos}

A adolescência é um período de mudanças e peculiaridades, intrínseco ao processo de formação do indivíduo. É denominado também, um estágio que engloba o desenvolvimento do autoconceito, autoestima e opiniões mais complexas, sendo as mudanças: hormonais, físicas (como o crescimento dos seios e do pênis) e psíquicas (como pensamentos abstratos). Além do adolescente se deparar com novas e estressantes situações, como o aumento de responsabilidades, exigências e modificações tanto sociais, como familiares, o desenvolvimento da autonomia e independência, a necessidade de se adaptar as novas propostas, onde os acontecimentos são frenéticos, efetivos e as diversidades dos modos de viver a vida. A entrada no universo adulto, muitas vezes causa dúvida, inquietações, incerteza, insegurança e mudanças de comportamento com os pares sociais; isto pode desencadear em outras comorbidades como: fobia, stress, alterações no sono e bulimia. (Aragão, Coutinho, Araújo, \& Castanha, 2007;
Melo \& Moreira, 2008; Valverde, Vitalle, Sampaio, \& Shoen, 2012).

Com o final da infância e a chegada da vida adulta, o adolescente se depara com um território completamente novo, ao mesmo tempo, em que é forçado a deixar o conforto de sua vida infantil. Por ser uma fase conturbada, há fatores que influenciam a sua manifestação, esses fatores estão facilmente interligados com o meio social em que o adolescente coabita $e$ as adversidades apresentadas nessa esfera, todavia, o meio não está totalmente conectado com os fatores psicossocial, assim dito por Antunes e Campos (2007). Em pesquisa realizada por Monteiro e Lage (2007) os autores afirmam que a depressão também é uma resposta a uma desorganização orgânica, onde o adolescente se encontra desamparado, por isso, os sintomas físicos ficam evidenciados. Atualmente, tem-se o conhecimento das causas e efeitos da depressão, especificamente, quando os sintomas são exibidos durante a adolescência.

Fukuda et al. (2016), corroboraram em sua pesquisa que os sintomas depressivos são normalmente apre- 
sentados entre os 10 a 13 anos de idade. Contudo, os comportamentos negativos nas relações sociais, perturbações do tipo externalizante e internalizante, principalmente depressão, ansiedade e agressividade, costumam ficar evidenciados em torno dos 11 a 16 anos de idade.

Pode se observar, que a estrutura emocional foi também critério de avaliação, como encontrados nos estudos realizados com os jovens de escolas públicas e privadas de uma cidade do interior do estado do Rio de Janeiro, os resultados revelaram a baixa autoestima, a não autodeterminação, não resiliência, falta de confiança em si e a insatisfação com a vida (Avanci, Assis, \& Oliveira, 2008).

Quanto a prevalência dos sintomas depressivos entre os sexos, a pesquisa realizada por Crivelatti, Durman e Hofstatter (2007) identificou no sexo feminino, estatisticamente destacado em porcentagens superiores, quando comparados ao sexo masculino à idade adulta, principalmente, ao crescente número de indivíduos que apresentam depressão. Tal fenômeno, se deve ao fato das adolescentes evidenciarem vulnerabilidade e passar por problemas psicológicos e sociais ainda na infância, visto que se preocupam com a aparência e sofrem com os padrões exigidos pela sociedade. Da mesma forma, Ferreira, Farias, \& Silvares (2010) pontuam que existem padrões definidos previamente condizentes e relacionados à hierarquia social, que por ser do sexo feminino, teriam menos oportunidades para o seu desenvolvimento profissional e individual. Além de estarem biologicamente destinadas a enfrentar processos hormonais de forma mais intensificada em relação aos meninos (Crivelatti, Durman, \& Hofstatter, 2007).

Aragão et al. (2009) citam sobre a diferença dos sintomas manifestados em mulheres, os autores destacam sentimentos de insignificância, tédio, irritabilidade, impaciência e inquietação, baixa autoestima e preocupação com a imagem que se é passada. Enquanto os homens relatam repulsa, provocação, arrogância e problemas de comportamento, como: não comparecimento às aulas, atos violentos e agressivos, furtos e abuso de substâncias psicoativas.

Assim, é necessário diferenciar os sintomas do transtorno depressivo e de uma tristeza; por este moti- vo, a identificação da depressão no adolescente se torna difícil. Melo e Moreira (2008) em suas descrições, enfatizaram algumas manifestações peculiares observadas no adolescente deprimido, como: a exaustão, linguagem arrastada, locomoção lenta, pensamentos de lamentação e ineficácia, visões pessimistas quanto ao futuro, pensamentos ou atos suicidas, automutilação, sono perturbado, diminuição no apetite, humor irritado, abuso de álcool, comportamentos de medo ou acentuando incômodo quando não obtém a atenção que desejam.

Da mesma forma, Aragão et al. (2007) corroboram com as experiências emocionais apresentadas pelos adolescentes, como, o choro frequente, interesse diminuído em atividades que antes eram prazerosas, energia física e mental diminuídas. Diante disso, percebe-se que a perda da vida infantil, a insegurança sobre o futuro e a não definição do seu papel na sociedade, faz com que o jovem se retraia. A falta de conhecimento da população sobre problemas mentais abre caminho para o surgimento de mitos e controvérsias, que dão origem ao preconceito com tais doenças. De acordo com as pesquisas de Fukuda et al. (2016), esse fator é apontado como uma das barreiras na procura de tratamento médico e que, ocasionalmente, resultam em um agravamento do estado clínico do paciente.

Avanci et al. (2008) além de constatarem-se características emocionais, também foram apresentadas características psicossociais, essas são separadas em fatores familiares e demográficos: falta de interação com os pais ou irmãos, problemas financeiros, problemas com álcool e drogas em geral, divórcio dos pais ou novos casamentos instituídos pelos genitores, violência com o adolescente ou entre os próprios pais, em alguns casos, além da agressão física, há a agressão sexual. Além de trazer consigo a fome, a falta de dinheiro e o acesso à educação, os indivíduos são levados a enfrentar problemas desde a tenra infância em casa.

A pesquisa realizada por Melo e Moreira (2008) aponta que os principais fatores que podem desencadear a síndrome depressiva em adolescentes, pelo discurso dos entrevistados, são os conflitos familiares, a situação social, o padrão de beleza, substâncias psicoativas, que delimitam as extremidades socioeconômicas e culturais, que os expõem 
as alternativas e limites de sua realidade. Observase que a família é o centro de investigação para identificar possíveis relações entre os vínculos instituídos com pais e filhos, tais como, a afetividade e o conflito familiar relacionado com a depressão com crianças e adolescentes, resultados sugerem que há associação (Teodoro, Cardoso, \& Freitas, 2009).

Corroborando os estudos de Abaid, Aglio e Koller (2010) que realizaram um estudo com crianças e adolescentes institucionalizados, com o objetivo de verificar as variáveis psicossociais dos sintomas nesse grupo estudado. $O$ resultado evidenciou que há necessidade de trabalhos interventivos nas escolas e nas famílias, a fim de ressaltar a importância desses modelos constituintes e denominados em redes de apoio.

Além disso, a mídia exerce um panorama, considerado como uma espécie de catalisador à depressão, onde a sociedade recebe muitas informações e padrões de vida, se deparando com influências de corpo e rostos perfeitos que acabam trazendo um sentimento de desconforto e fracasso pelos adolescentes (Melo \& Moreira, 2008). Percebeu-se que - adolescente pode desenvolver alguns sentimentos negativos para si, como uma baixa autoestima, falta de determinação e de confiança em si, se tornando desprotegido para a síndrome depressiva. A fantasia ligada ao corpo e a vida perfeita são transmitidas pela mídia, televisão ou revistas de estética, que influenciam diretamente e indiretamente, contribuindo para o desenvolvimento de cobranças internas e buscas insensatas, causando sofrimento psíquico.

Refletindo sobre o aspecto sócio demográfico no Brasil há uma considerável evasão escolar, indicando alguns dos motivos desses jovens de não permanecerem na escola, pois, muitos desde o início da adolescência são impulsionados a ingressar no mercado de trabalho e assim, ajudar na renda familiar. Devido à falta de orientação, os púberes podem ficar vulneráveis a prostituição e a delitos, ficando suscetíveis ao contato com as drogas e doenças sexualmente transmitidas, podendo este ser um dos motivos para o grande aumento do índice de depressão em indivíduos cada vez mais jovens (Melo \& Moreira, 2008). Corroborando com os estudos, Souza et al. (2008) pontuam que há fatores como, fracasso escolar, renda económica baixa e abuso de álcool são indicativos para o transtorno de conduta. Já a vulnerabilidade social foi destaque na pesquisa de Barbosa et al. (2016) destacando o tempo que os jovens ficam na internet, televisão e computador demostrando um alto indicador para o transtorno depressivo, principalmente nos meninos.

A instituição escolar é um agente facilitador que poderá agir de modo a identificar possíveis alterações no comportamento de seus alunos e alertar os responsáveis, para a procura de ajuda especializada. Desta forma, favorecerá a busca pela saúde mental. Sendo assim, é necessário que a tríade escola - família - educadores contribuam na prevenção, e apresentar em seu contexto escolar um profissional capaz de responder as perguntas dos adolescentes relacionadas ao assunto (Aragão et al., 2007; Ribeiro, Coutinho, Nascimento, 2010). A falta de informação sobre a depressão acaba contribuindo para o agravamento da saúde do adolescente, sendo vista apenas como algo passageiro e comum na adolescência, trazendo uma dificuldade para o diagnóstico. Portanto, a escola é um agente facilitador para identificar sobre a possibilidade de o adolescente estar em sofrimento psíquico e assim auxiliar com os responsáveis do adolescente.

Esse profissional especializado necessita de aprimoramento e entendimento sobre o desenvolvimento emocional e biológico das diferentes idades, sobretudo, ser compreensivo as dificuldades do aluno, compartilhando a responsabilidade com todos que formam a rede de proteção e de cuidados com o adolescente depressivo. (Valverde et al., 2012). Além disso, a carência de conhecimento sobre a depressão acaba contribuindo para o agravamento da saúde do adolescente, sendo vista apenas como algo passageiro e comum na adolescência, trazendo uma dificuldade para o diagnóstico. Portanto, a escola é um agente facilitador para identificar comportamentos e sintomas atrelados ao sofrimento psíquico, sendo uma unidade social fundamental para o auxílio junto aos responsáveis do aluno.

\section{Intervenções recebidas: antes e depois do trata- mento ambulatorial}

Conforme visto na pesquisa de Antunes e Campos (2007) os autores apresentam-nos uma espécie de 
"rede terapêutica", que se compõe antes mesmo da procura por atendimento especializado. Esta rede é formada por um circulo de aconselhamento de familiares, amigos, vizinhos, podendo haver também, uma procura por curandeiros ou outros aspectos ligados à religião do adolescente e da família, aproximação esta que poderá ser motivada pela própria doença. Com o mesmo cunho Aragão et al. (2009) realizaram uma pesquisa com os alunos do ensino médio, que nos dita outro tópico contribuinte para o tratamento, o apoio religioso.

A partir do estudo de Fukuda et al. (2016) existem três principais barreiras para a procura de tratamento médico, tais como, as barreiras estruturais, que estão relacionadas com o transporte e a disponibilidade de dinheiro para pagar o tratamento, as barreiras referentes a percepção dos problemas mentais e a ideia de que é possível se curar sem tratamento adequado e por fim, barreiras que se relacionam ao preconceito e falta de boas experiências com serviços de saúde em casos anteriores.

Diagnosticado a depressão no adolescente é importante frisar que em algumas famílias, a busca por ajuda profissional têm acarretado no distanciamento do preconceito ao tratamento e também, a aceitação da doença pelo paciente e o desejo de melhora, fatores importantes para uma recuperação satisfatória (Valverde et al., 2012). O meio social que o adolescente está inserido, nesse caso, a escola é fator determinante frente à percepção dos sintomas evidenciados por esses alunos, assim, percebe-se a ampliação para a conduta necessária dos profissionais envolvidos, pois, acaba sendo um auxilio para os pais na identificação do problema. (Jatobá \& Bastos, 2007)

Da mesma forma, considera-se fundamental que médicos e profissionais da saúde atentem para os adolescentes, sobre o uso exagerado das redes sociais, ajudando e alertando a família a perceber o que esse adolescente vivencia do mundo real e virtual (Moutinho et al., 2013)

A pesquisa de Fukuda et al. (2016) levantou algumas das dificuldades que os pacientes adolescentes têm de enfrentar durante o tratamento e as descri- tas com maior frequência são questões relacionadas com a escola, além dos enfrentamentos da ansiedade, da depressão e do medo. Logo em seguida, há problemas comportamentais e de convívio sociais, seja com a família ou com os amigos. Percebe-se que os mesmos podem não receber o tratamento médico adequado e está propensa a queda de rendimento escolar, uso de substâncias psicoativas, agressão e envolvimento em atos criminosos. Constantemente, há propagandas e anúncios sobre os medicamentos antidepressivos. Esses são vendidos pela mídia como promessa de uma vida tranquila e a garantia de harmonia. Assim, o consumismo associado ao uso abusivo e inadequado de medicamentos para o alívio da dor emocional, agregado ao preconceito com tais doenças psíquicas, faz com que sejam vistos pela população, como uma cura para o enfermo ou apenas a prevenção de uma depressão futura. (Monteiro \& Lage, 2007).

As contribuições deste estudo para a área da saúde, resulta da síntese dos resultados discutidos nos manuscritos estudados, principalmente, acerca dos fatores psicossociais associados aos adolescentes depressivos e conjuntamente sobre as intervenções recebidas. Esta síntese expõe com o intuito de favorecer e prover uma discussão, frente ao transtorno depressivo na adolescência e o manejo adequado para a reabilitação da saúde mental.

\section{Considerações finais}

Assim, na compreensão do conteúdo dos artigos analisados, consideramos que o processo de entendimento sobre o meio social em que o adolescente está inserido é considerado um fator de impacto que reflete não somente no âmbito familiar, mas também, na escola e na sociedade em geral. Percebeuse que a possibilidade de ter um filho adolescente com alguma doença mental, afeta profundamente a família, que geralmente se sente angustiada e confusa por não encontrar recursos para lidar diante a situação de sofrimento, além de enfrentar o preconceito existente na sociedade e até mesmo no próprio meio familiar. 
Observou-se uma espécie de rede terapêutica ou rede de proteção, o que facilita e contribui para o tratamento; esta rede é formada pelos amigos, a família do adolescente e o próprio tratamento médico em que ele está envolvido, em alguns casos, a religião do paciente também é considerada como sendo, um fator importante para o processo. Entretanto, existem barreiras que dificultam a profilaxia, dentre elas, podemos citar a baixa renda familiar, o preconceito e o desconhecimento sobre a depressão, a espera demasiada para uma consulta ambulatorial e os recursos escassos que ainda podem refletir no atendimento clínico, como, falta de medicamento e mesmo longas horas de espera.

Destarte, a vigente pesquisa obteve o objetivo apontado, de investigar e explorar estudos primários, tendo em vista que o interesse em compreender como esses resultados desta revisão integrativa da literatura nacional, direcionam a necessidade de uma discussão mais profunda e intimista entre escola e pais, sobre a saúde mental de seus filhos, assuntos esses que poderão ser discutidos em forma de palestras com pais e responsáveis dos adolescentes, bem como profissionais preparados para perceberem comportamentos sintomatológicos em seus alunos, fazendo com que o diagnóstico e o processo de encaminhamento ambulatorial possa ocorrer com facilidade. Evidencia-se o olhar de acolhimento dos pais e familiares na percepção dos sintomas depressivos apresentados pelos filhos, diferenciando de aspectos comportamentais inerentes aos adolescentes.

\section{Contribuições os autores}

Lima, G. M. P. A. foi responsável pela escolha do tema; busca e análise dos dados da pesquisa; interpretação dos resultados ; redação e revisão crítica do conteúdo do artigo científico. Mio, J. V. R. foi responsável pela busca e análise dos dados da pesquisa e redação; Santos, G. N. foi responsável pela busca e análise dos dados da pesquisa e redação; Campos, C. J. G. foi responsável pela escolha do tema; análise dos dados e interpretação dos resultados.

\section{Conflitos de interesses}

Nenhum conflito financeiro, legal ou político envolvendo terceiros (governo, empresas e fundações privadas, etc.) foi declarado para nenhum aspecto do trabalho submetido (incluindo mas não limitandose a subvenções e financiamentos, conselho consultivo, desenho de estudo, preparação de manuscrito, análise estatística, etc).

\section{Referências Bibliográficas}

Antunes, H. M., Campos, C. J. G., Lima, G. M. P. A., \& Ferraz, I. L. G. (2016). Motivos e crenças de familiares frente ao tratamento do transtorno depressivo na infância: Estudo qualitativo. Estudos de Psicologia, 21 (2), 157-166. Recuperado de http://www.scielo.br/pdf/ epsic/v2 ln2/1413-294X-epsic-21-02-0157.pdf. doi: $10.5935 / 1678-4669.20160016$

Bardin, L. (201 1). Análise de Conteúdo. São Paulo: Edições 70.

Chavez, E. R. (2014). Transtornos depressivos em niños y adolescentes. Diagnostico, 53(3),136-141. Recuperado de http://pesquisa.bvsalud.org/portal/resource/pt/ lil-765154

Crujo, M., \& Marques, C. (2009). As perturbações emocionais - ansiedade e depressão na criança e no adolescente. Rev Port Clin Geral, 25, 576-82. Recuperado de http://www.rpmgf.pt/ojs/index.php/rpmgf/article/ viewFile/10675/10411

Encarnação, R., Moura, M., Gomes, F., \& Silva, P. C. (2011). Caracterização dos casos observados numa equipe de psiquiatria da infância e adolescência: Um estudo retrospectivo. Acta Med Port, 24(6), 925-934.

Schoen-Ferreira, T. H., Aznar-Farias, M., \& Silvares, E. F. M. (2010). Adolescência através dos Séculos. Psicologia: Teoria e Pesquisa, 26(2), 227-234. Recuperado de http://www.scielo.br/pdf/ptp/v26n2/a04v26n2.pdf. doi: $10.1590 /$ S0102-37722010000200004

Melo, A. K., Siebra, A. J., \& Moreira, V. (2017). Depressão em Adolescentes: Revisão da Literatura e o Lugar da Pesquisa Fenomenológica. Psicologia Ciência e Profissão, 37(1), 18-34. Recuperado de http://www.scielo.br/pdf/pcp/v37n 1 / 19823703-pcp-37-1-0018.pdf. doi: 10.1590/1982$\underline{37030001712014}$

Moher, D., Liberati, A., Tetzlaff, J., Altman, D. G., The PRISMA Group. (2009). Preferred reporting items for systematic reviews and meta-analyses: the PRISMA Statement. PLoS Med, 6(6), e 1000097. Recuperado de https://www.ncbi.nlm.nih.gov/pubmed/19621072. doi: $10.1371 /$ journal.pmed.1000097

Resende, C., Santos, E., Santos, P., \& Ferrão, A. (2013). Depressão nos adolescentes - mito ou realidade?. Nascer e Crescer, 22(3), 145-150. Recuperado de http://www.scielo.mec.pt/pdf/nas/v22n3/v22n3a03. pdf 
Rojas, G., Gaete, J., Guajardo, V., Martínez, V., Barroilhet, S., Meneses, J., ... Araya, R. (2012). Consumo de substancias y sintomas depressivos em escolares de liceos municipales de Santiago. Rev Med Chile, 140, 184-191. Recuperado de https://scielo.conicyt.cl/ $\mathrm{pdf} / \mathrm{rmc} / \mathrm{v} 140 \mathrm{n} 2 /$ art06.pdf. doi: $10.4067 / \mathrm{s} 0034$ 98872012000200006

Sobral, F. R., \& Campos, C. J. G. (2012). Utilização de metodologia ativa no ensino e assistência de enfermagem na produção nacional: revisão integrativa. Revista da Escola de Enfermagem da USP, 46(1), 208-218. Recuperado de http://www.scielo. $\mathrm{br} / \mathrm{pdf} / \mathrm{reeusp} / \mathrm{v} 46 \mathrm{n} 1$ /v46n l a28.pdf. doi: 10.1590/ S0080-62342012000100028

Souza, M. T., Silva, M. D., \& Carvalho, R. (2010). Revisão Integrativa: o que é e como fazer. Revista Einstein, $8(1), 102-6$. Recuperado de http://www.scielo.br/ pdf/eins/v8n1/pt_1679-4508-eins-8-1-0102.pdf. doi: $\underline{10.1590 / \mathrm{s} 1679-45082010 \mathrm{rw} 1134}$

Whittemore, R., \& Knalf, K. (2005). The integrative review: updated methodology. J Adv Nurs, 52(5), 54653. Recuperado de https://www.ncbi.nlm.nih. gov/pubmed/16268861. doi:10.1111/i.1365$\underline{2648.2005 .03621 . x}$

World Health Organization. (2017). Depression and Other Common Mental Disorders: Global Health Estimates. Geneva: WHO.

Xerinda, J. E., \& Bucher-Maluschke, J. S. N. F. (2016). Estrutura Familiar de um Adolescente com Depressão atendido no Centro de Reabilitação Psicológica Infantil e Juvenil de Maputo Moçambique. Adolesc Saúde, 13(3), 16 24. Recuperado de http://www.adolescenciaesaude. com/detalhe_artigo.asp?id=602 